\title{
Optimizing the mass transport of wall-tube electrodes for protein film electrochemistry
}

\author{
Asmaa Hadj Ahmed ${ }^{\mathrm{a}, \mathrm{b}}$, Jean-Vincent Daurelle ${ }^{\mathrm{b}, *}$, Vincent Fourmond ${ }^{\mathrm{a}, *}$ \\ ${ }^{a}$ Aix-Marseille Université, CNRS, BIP UMR 7281, 31 Chemin J. AIGUIER, CS70071, F-13402 Marseille Cedex 20, (France) \\ ${ }^{b}$ Laboratoire IUSTI (UMR AMU-CNRS 7343) Polytech Marseille, Dpt Mécanique Energétique (ME), Technopôle de Chateau Gombert, 5 rue Enrico Fermi, 13453 \\ Marseille cedex 13, France
}

\begin{abstract}
Protein Film Electrochemistry (PFE) is a technique in which an enzyme is directly wired to an electrode and its catalytic turnover rate is measured under the form of an electrical current. This technique has proved useful for the study of a number of enzymes, but requires fast transport of the enzymatic substrate towards the electrode. In a previous work (Fadel et al, Phys. Chem. Chem. Phys., $2019,21,12360$ ), we have proposed a new design based on the wall-tube electrode that provides better transport than the rotating disc electrode, which is usually employed for PFE studies. In the present work, we use computational fluid dynamics to explore the effects of the various parameters of the cell, and propose a semi-empirical formula suitable to predict the mass-transport coefficient and the wall shear stress on the electrode. We use a 3D-printed cell to experimentally validate our predictions.
\end{abstract}

Keywords: hydrodynamic electrode; wall-tube; computational fluid dynamics; protein film electrochemistry

\section{Introduction}

Protein Film Electrochemistry (PFE) is an electrochemical technique that is used for studying the mechanism of redox enzymes. It consists in immobilizing a film of enzymes on an electrode in a configuration where the electron transfer is direct, and measuring the response in current to changes in conditions $[1,2]$. The current generated from the exchange of electrons between the enzymes and the electrode is proportional to the turnover rate of the enzyme, which makes it possible to learn about various aspects of the reactivity of the immobilized enzyme [3-5]. As the enzymatic substrate is consumed at the electrode by the catalytic reaction, PFE requires fast transport of the substrate towards the electrode. Sometimes, the enzymatic reaction is slow enough that passive diffusion suffices [6]; however, most enzymes require faster transport, which is usually provided by using a rotating disc electrode (RDE) as working electrode [7]. However, our group has recently reached a limitation of the RDE setup while working on CO dehydrogenases, the enzymes which catalyze the reversible oxidation of $\mathrm{CO}$ to $\mathrm{CO}_{2}$ [8]: these enzymes are so fast that even it the highest practical rotation rates, mass-transport of $\mathrm{CO}$ is limiting $[9,10]$, which greatly complicates the study of this enzyme.

To overcome this limitation, we have chosen to design a new electrochemical cell with improved transport properties. The mass transport should be as homogeneous as possible, to avoid heterogeneity of substrate concentration on the electrode, which would greatly complicate the studies as the enzymatic response is highly non-linear in function of the substrate concentration. This property is known as "uniform accessibility" [11]. Moreover, since the cell will be used by immobilizing

\footnotetext{
${ }^{*}$ Corresponding authors: jean-vincent.daurelle@univ-amu.fr and vincent.fourmond@imm.cnrs.fr.
}

enzymes on the surface of the working electrode, a particular interest should be given to the forces applied by the fluid at the surface (the shear stress) since it could be one of the main reasons for the desorption of the film of enzyme [12,13]. Jet electrodes, in which the flow impinges normally from an inlet toward a disk electrode[14], are good candidates for solving the transport limitation since they ensure high and controllable mass transport[15]. The jet electrodes can be divided into two sub-types depending on the size of the electrode with respect to the size of the inlet: the "wall jet" configuration[16], in which the diameter of the inlet is smaller then the one of the electrode, which is widely applied for electrochemical detection in liquid chromatography [17, 18], and the "wall tube" configuration[19], in which the electrode is smaller than the inlet. Only the latter provides uniform accessibility[20, 11].

After an initial screening of a number of geometries using computational fluid dynamics, we have recently proposed a design based on wall-tube electrodes[21], which provides a factorof-three improvement with respect to the classical RDE setup (assuming a rotation rate of $5000 \mathrm{rpm}$ ). However, in our previous work, we relied only on computational fluid dynamics simulations to predict the mass transport in given conditions. This contrasts with the ease of use of the RDE, for which a reliable analytical formula is available for predicting the mass transfer properties[22]. Hydrodynamics in impinging jets have been addressed by many studies in mass and in heat transfer [23, 24]. Homann and coworkers developed a mathematical solution to describe the boundary layer flow in the stagnation region of a uniform flowing field on an infinite plate [25]. This solution was extended to the case of a uniform impinging jet, where it was proved that a boundary layer of a constant thickness is present in the stagnation region. Chia and coworkers demonstrated that the mass transfer rate at the impinged surface is rel- 


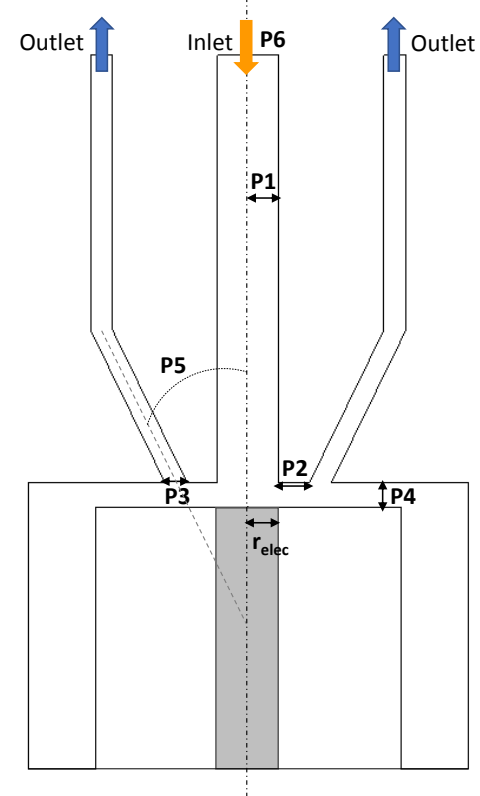

Figure 1: Schematic representation of the geometry of the cell, indicating the 6 parameters varied in the systematic approach. The detailed meaning and values of the parameters are given in table 1

atively uniform in the case of wall-tube electrodes[26]. Semiempirical solutions of mass transport in a uniform impinging jet were developed in previous studies [27-29, 11] but they were limited to certain configurations. In spite of the amount of studies about the mass transport in jet electrodes, there were only few that addressed shear stress.

In this work, we have developed semi-empirical formulas suitable to predict the mass-transport properties and the shear stress for wall-tube cells such as the one we have previously proposed[21]. We have used an approach based on computational fluid dynamics, using first a systematic sensitivity study to determine the most influencing parameters, and then focusing on them to propose an analytical formula that reproduces our data within $10 \%$. We then demonstrate the validity of our formulas by verifying them experimentally using the reduction of ferricyanide in a 3D-printed cell.

\section{Results}

Figure 1 represents a schematic view of the experimental setup, similar to the one we have used in our previous work[21]. The fluid comes through the inlet in the center of the scheme (orange downward arrow), impinges on the working electrode (the gray area at the bottom) and is evacuated via symmetric outlets that are initially inclined with respect to the vertical axis (blue upward arrows). The reference and counter electrodes are near the working electrode (they are not represented in figure 1). The cell is designed in two parts, one with the inlet and outlets, and the other with the electrodes. For more detailed information, see ref. 21 .
Numerical simulations were performed using commercial finite volumes modelling package starccm + from CD Adapco to study the flow and transport properties of the cells in silico. We used a physical model that is based on a laminar incompressible flow at steady state regime in a $2 \mathrm{D}$ axis symmetric domain with a mesh of a base size of $50 \mu \mathrm{m}$. The size of the mesh cells decrease when approaching the working electrode so we can properly model the strong gradient of concentration in this zone (diffusion layer). The boundary conditions that were used are as following: we imposed a given velocity and a fixed concentration in the inlet of the cell, a split value condition at the outlets, a non slip condition on the walls. At the working electrode, we assumed an infinitely fast catalytic reaction that consumes all the reaction's substrate, so its concentration is zero at the surface of the electrode. More details about the meshing and the boundary conditions can be found in supplementary section $\mathrm{S} 1$.

These simulations were used to compute the velocity profiles in order to determine the value of the wall shear-stress at the electrode, but, most importantly, to compute the flux of electroactive species towards the electrode, in order to determine the mass-transport coefficient $m$, defined by:

$$
j=m \times(c(\infty)-c(0))
$$

in which $j$ is the flux of species at the electrode, $c(\infty)$ the concentration of species in the upstream flow and $c(0)$ the concentration of species at the electrode (in our case, $c(0)=0$ ).

In contrast to our previous work[21], we simplified the 3D design into a $2 \mathrm{D}$ design with a rotational invariance, which provided both finer grained results, less discretization artifacts, and faster computation time. We show in supplementary section S2 (and figure S3) that the two models predict the same flux within less than $10 \%$ for values of $r$ between 0 and at least the value of the inlet radius.

\subsection{Sensitivity study}

In a first part, we studied the influence of the design parameters of the setup on the properties of the cell. We chose to focus on six parameters (figure 1): P1, the inlet radius, P2, the distance between the center of the cell and the center of the outlets, P3, the diameter of the outlets, P4, the distance between the inlet and the electrode, P5, the angle of the outlets, and P6, the input flow rate. The electrode radius plays also a very important role in the properties of the setup, but as it can be varied at will by just changing the size of the region in which the flux is considered (see below), we haven't included it as a parameter.

We used a design of experiments approach to systematically study the influence of these six parameters on the masstransport properties of the cell. We used a full-factorial design with two levels[30], which consists in defining a "low" and a "high" level for all the parameters and running simulations for all the possibilities (here, $2^{6}=64$ combinations). For the levels, we used values that correspond to the limits of what we could experimentally build (see table 1 ).

We ran the 64 simulations, extracted the mass-transport coefficient (equation (1)) and applied the statistical treatment described in ref. 30. The results of this treatment are "effects" 


\begin{tabular}{|c|c|c|c|c|c|c|}
\hline Parameter & $\begin{array}{c}\text { Inlet radius } \\
\text { (P1) } \\
\mathrm{mm}\end{array}$ & $\begin{array}{c}\text { Distance inlet-outlet } \\
\text { (P2) } \\
\mathrm{mm}\end{array}$ & $\begin{array}{l}\text { Outlet thickness } \\
\text { (P3) } \\
\text { mm }\end{array}$ & $\begin{array}{c}\text { distance nozzle-electrode } \\
\text { (P4) } \\
\mathrm{mm}\end{array}$ & $\begin{array}{l}\text { inlet-outlet angle } \\
\text { (P5) } \\
\circ\end{array}$ & $\begin{array}{c}\text { Flow rate } \\
\text { (P6) } \\
\mathrm{mL} / \mathrm{min}\end{array}$ \\
\hline Low level & 0.1 & 0.05 & 0.1 & 0.1 & 10 & 1 \\
\hline High level & 0.5 & 1 & 1 & 0.5 & 80 & 5 \\
\hline
\end{tabular}

Table 1: Values of the low and high levels of the parameters varied in the sensitivity studies. The definition of the parameters is in figure 1.

\begin{tabular}{cc}
\hline Parameters & Effects $\left(\mathrm{mm} . \mathrm{s}^{-1}\right)$ \\
\hline Inlet radius (P1) & -1.26953 \\
Distance inlet-outlet (P2) & 0.00194 \\
Outlet thickness (P3) & 0.00509 \\
Distance nozzle-electrode (P4) & -0.11021 \\
Inlet-outlet angle (P5) & -0.00073 \\
Flow rate (P6) & 0.58171 \\
\hline Average & 0.73959 \\
\hline
\end{tabular}

Table 2: The single effects of all parameters on the simulated mass transport coefficient from the sensitivity study for $r_{\text {elect }}=0.1 \mathrm{~mm}$. Average is the average of the mass-transport coefficient over all the 64 simulations. The list of all the effects including multi-parameter changes is found in supplementary table S1 (section S3).

for each parameter (and combination of parameters), which are the difference between the average values of all the "high level" simulations and the average values of the "low level" simulations. The greater the effect (in absolute value), the more impact the parameter has on the mass transport. Table 2 lists all the single-parameter effects. It shows that, out of the six parameters, only three have a significant influence: P1, P4 and $\mathrm{P} 6$, respectively the inlet radius, the inlet-to-electrode distance and the flow rate (the full list of effects, including the effect of jointly changing two or more parameters can be found in supplementary Table S1, section S3). The inlet radius has a strong negative effect, the flow rate a slightly smaller positive effect, and the inlet-electrode distance has about 10 times less effect as the inlet radius. The effects of the other parameters, are at least one order of magnitude smaller than that of P4. These results are in line with other works, which found that the inlet diameter, the flow rate and the distance between inlet and electrode were the most important parameters[16, 29].

\subsection{Derivation of semi-empirical formulas}

In a second step, we proceeded to propose semi-empirical formulas to predict the values of the mass-transport coefficient and the wall shear-stress, restricting ourselves to the dependence on the most influencing parameters determined in the first step.

Theoretical background: the hydrodynamic constant. We consider a stationary incompressible flow. According to Homann [25], the axial $(v)$ and the radial $(u)$ velocities in the stagnation region can be written under the following forms:

$$
\begin{aligned}
& u=\operatorname{ar} \phi^{\prime}(\eta) \\
& v=-2 \sqrt{a v} \phi(\eta)
\end{aligned}
$$

in which $a$ is a constant, called the "hydrodynamic constant" (of dimension the reciprocal of time), $\eta$ is the dimensionless axial coordinate, and $\phi(\eta)$ is a function. $\eta$ is defined as:

$$
\eta=z \sqrt{a / v}
$$

where $v$ is the kinematic viscosity.

Frossling proposed a Taylor expansion for the function $\phi(\eta)$ [31]. For the purposes of this article, we have truncated the expansion to its first term[29]:

$$
\phi(\eta)=0.656 \times \eta^{2}
$$

This expression is highly general since it has been applied to wall-jet cells[29] but it is also valid in the case of the rotating disc electrode. We first demonstrate that the knowledge of the value of $a$ is sufficient to determine the mass-transport coefficient and the shear stress; we then focus on extracting the values of $a$ from the simulations and deriving an empirical formula for the prediction of $a$. Note that the actual value of the coefficient in equation (4) has little importance here, since changing the coefficient is equivalent to changing the value of $a$; we have used 0.656 to be consistent with previous studies[31].

We now compute the concentration profile $c$ of the electroactive species in the solution. The electroactive species is consumed at the electrode, with a rate supposed to be sufficiently fast that $c=0$ at the electrode surface. Similarly to what has been done before[29], we assume that the concentration is independent of the radial coordinate, so that $c$ is only a function of $\eta$. Under this hypothesis, and using the expression of the velocities in (2), the steady-state convection-diffusion equation for $c$ can be expressed as:

$$
\frac{\mathrm{d}^{2} c}{\mathrm{~d} \eta^{2}}+2 S c \phi(\eta) \frac{\mathrm{d} c}{\mathrm{~d} \eta}=0
$$

where we have introduced the Schmidt number,

$$
S c=v / D
$$

Double integration of (5) yields:

$$
\frac{c(\infty)-c(0)}{\kappa_{0}}=\int_{0}^{\infty} \exp (-2 S c \Phi(\eta)) \mathrm{d} \eta
$$


in which $\kappa_{0}$ is an integration constant (the value of the first derivative of $c$ at 0$), c(0)$ is the concentration at the electrode, $c(\infty)$ at a large distance from the electrode (in the bulk), and $\Phi$ is a primitive of $\phi$ defined by:

$$
\Phi(\eta)=\int_{0}^{\eta} \phi\left(\eta^{\prime}\right) \mathrm{d} \eta^{\prime}
$$

Equation (1) for the mass-transport coefficient $m$ can be rewritten thus:

$$
D \frac{\mathrm{d} c}{\mathrm{~d} z}=m \times(c(\infty)-c(0))
$$

Integrating of (7) using the expression of $\phi(\eta)$ given in (4) and injecting the result into equation (9) yields:

$$
m=\beta a^{1 / 2} D^{2 / 3} v^{-1 / 6}
$$

where $\beta=0.85002$. Note that equation (10) is formally identical to the equation giving the mass transport coefficient for a rotating disc electrode, if one uses $a=0.533 \times \omega$, in which $\omega$ is the angular velocity of the RDE.

The wall shear stress $\tau_{w}$ is defined as:

$$
\tau_{w}=\rho v \times\left.\frac{\mathrm{d} u}{\mathrm{~d} z}\right|_{z=0}
$$

Using the expressions of $u$ and $\phi(\eta)$ from equations (2) and (4), we obtain:

$$
\tau_{\mathrm{w}}=1.312 \times \rho v^{1 / 2} a^{3 / 2} r
$$

Equations (12) and (10) show that it is possible to deduce $m$ and $\tau_{w}$ from the value of the hydrodynamic constant $a$.

Determination of the hydrodynamic constant as a function of the parameters. With the aim of determining the dependence of the hydrodynamic constant $a$ on the flow rate $Q_{v}$, the inlet diameter $r_{j e t}$ and $h$ the distance nozzle-electrode, we ran a series of $6 \times 6 \times 3=108$ simulations with all the possible combinations of the parameter values listed in table 3 . For all these simulations, we have extracted the value of $a$ by fitting either equation $(2 a)$ or $(2 b)$ to the values of the velocities in a neighbourhood of the electrode (see supplementary figures S4 and S5 in supplementary section S4 for more information). Figure 2 shows the 108 values of $a$, plotted as a function of the inlet radius $r_{\text {jet }}$.

The data show a clear decrease of $a$ as $r_{j e t}$ increases, along with a strong increase as $Q_{v}$ increases (the different values of $Q_{v}$ are distinguished by the shape of the points). The dependence on $h$ is less marked, but it is nevertheless visible that a decrease in $h$ leads to a slight increase in $a$.

To remove the largest part of the influence of the parameters on $a$, we have worked on a dimensionless hydrodynamic constant $a^{*}$ defined this way:

$$
a^{*}=\frac{a \pi r_{j e t}^{3}}{Q_{v}}
$$

We consider here that the hydrodynamic constant only depends on four dimensional parameters: $r_{j e t}, Q_{v}, h$, and the kinematic

\begin{tabular}{ccc}
\hline $\begin{array}{c}\text { Inlet radius } \\
r_{j e t} \\
(\mathrm{~mm})\end{array}$ & $\begin{array}{c}\text { Inlet-electrode distance } \\
h\end{array}$ & $\begin{array}{c}\text { Inlet flow rate } \\
Q_{v} \\
(\mathrm{~mm})\end{array}$ \\
\hline 0.25 & 0.15 & 1 \\
0.30 & 0.20 & 5 \\
0.35 & 0.25 & 10 \\
0.40 & 0.30 & \\
0.45 & 0.35 & \\
0.5 & 0.40 & \\
\hline
\end{tabular}

Table 3: Values of the different parameters used for the determination of the hydrodynamic constant. All the $6 \times 6 \times 3=108$ combinations were used.

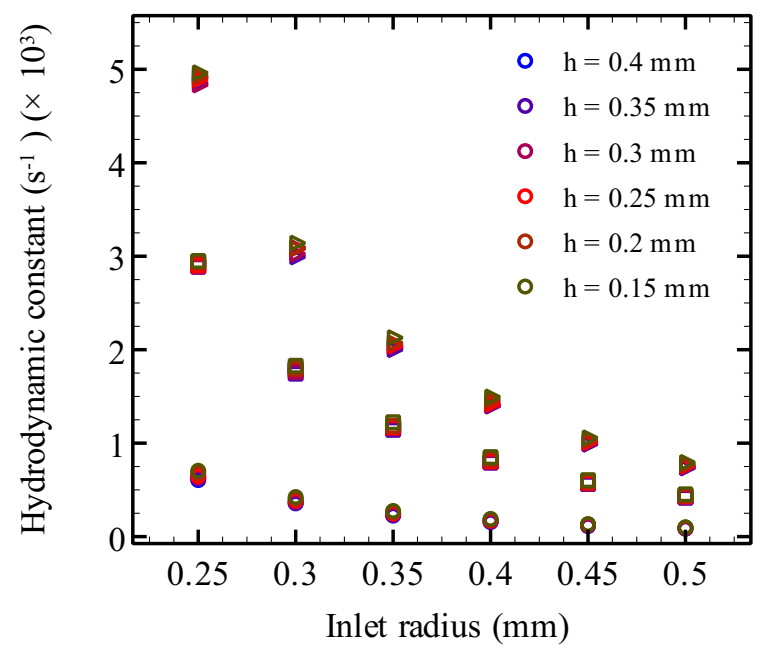

Figure 2: Values of the hydrodynamic constant $a$ determined from the simulations for the 108 combinations of parameters from table 3 , as a function of the radius of the inlet, $r_{j e t}$. The different symbol shapes correspond to different values of $Q_{v}: 1 \mathrm{~mL} / \mathrm{min}$ for circles, $5 \mathrm{~mL} / \mathrm{min}$ for squares and $10 \mathrm{~mL} / \mathrm{min}$ for triangles. The different values of $h$ are color-coded, as indicated in the graph itself. The other parameters were: distance inlet-outlet $(P 2=1 \mathrm{~mm})$, outlet thickness $(P 3=0.36 \mathrm{~mm})$, inlet-outlet angle $\left(P 5=26^{\circ}\right)$. The value of the electrode radius has no influence on the hydrodynamic parameters.

viscosity $v$, that have two independent dimensions in total (time and distance). The $\pi$ theorem therefore states that it can be expressed as a function of two independent dimensionless parameters. We have chosen the following:

$$
\alpha_{1}=\frac{r_{j e t}}{h} \quad \alpha_{2}=\frac{Q_{v}}{v h}
$$

We sought an approximation for $a^{*}$ in the form of a simple power law:

$$
a^{*}=\sigma \times \alpha_{1}^{\beta_{1}} \times \alpha_{2}^{\beta_{2}}
$$

in which $\sigma, \beta_{1}$ and $\beta_{2}$ are constants to determine. We used a simple systematic search to find the values of $\sigma, \beta_{1}$ and $\beta_{2}$ that yield the minimum root mean squared difference between the predicted and measured values of $a^{*}$. We found:

$$
\sigma=3.04993 \quad \beta_{1}=0.1685 \quad \beta_{2}=-0.0995
$$

Figure 3 represents the values of the $a^{*}$ predicted from equation (15) with the coefficients in (16) as a function of the values 


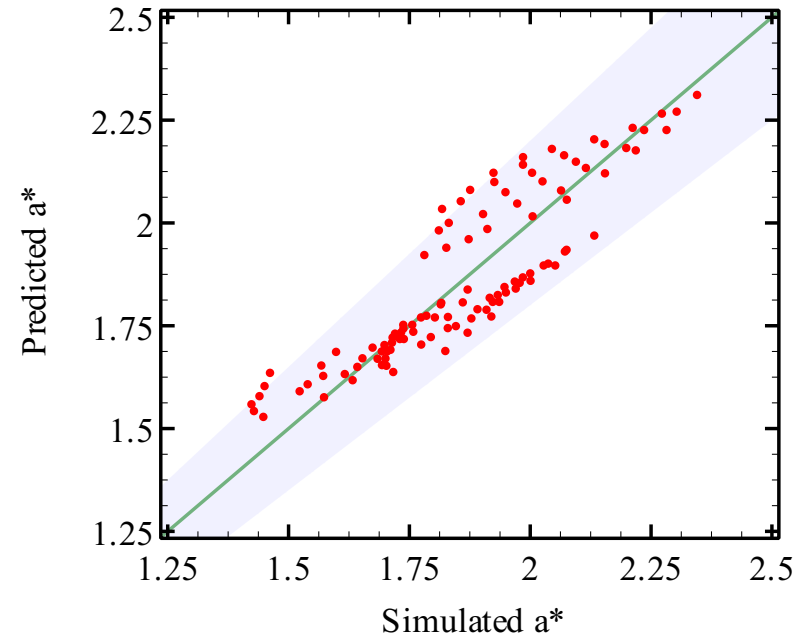

Figure 3: Values of $a^{*}$ predicted from equation (15) as a function of the value of $a^{*}$ determined from the simulations. The line corresponds to $y=x$. The shaded region corresponds to $y=x \times(1 \pm 0.1)$.

of $a^{*}$ determined from the simulations. The data show that all the points from the simulations lie in the $10 \%$ region on either side of the $y=x$ line, showing that the formula can predict hydrodynamic constants with a high accuracy.

Expressions for the mass-transport coefficient and the shear stress. Substituting (15) back into equations (10) and (12) yields the following expressions:

$$
\begin{aligned}
m & =0.83753 D^{2 / 3} v^{-0.11692} Q_{v}{ }^{0.45025} h^{-0.0345} r_{j e t}{ }^{-1.41575} \\
\tau_{w} & =1.255 \rho v^{0.64925} Q_{v}{ }^{1.35075} h^{-0.1035} r_{j e t}{ }^{-4.24725} r
\end{aligned}
$$

Figures 4 and supplementary figure S6 show that the above expressions correctly predict the values of $m$ and of $\tau_{w}$ deduced from the simulations. While this is not surprising, considering that the values of $m, \tau_{w}$, and $a$ are all deduced from the same velocity profiles, the quality of the predictions confirm the derivations above, and show that the semi-empirical formulas (17) reliably predict the results of our $2 \mathrm{D}$ simulations. The dependency on $a$ of equations (10) and (12) imply that a $10 \%$ error on $a$ corresponds to a $5 \%$ error on $m$ and a $15 \%$ error on $\tau_{w}$.

\subsection{Homogeneity of the mass transport}

For the cell to be used for electrochemical studies of enzymes, it is important that the flux is homogeneous, so that the concentration of the enzymatic substrate at the electrode is homogeneous[21]. In the simulations, we have used a very large electrode surface $\left(r_{\text {elec }}=1.25 \mathrm{~mm}\right)$ over which we assumed $c=0$. This made it possible to systematically probe the flux over a large surface and determine the maximum radius of the electrode which receives a homogeneous flux. We assume that electrode parts further away from the axis cannot influence the flux in electrode parts closer to the axis. This assumption is compatible with the direction of the flow, since it seems very

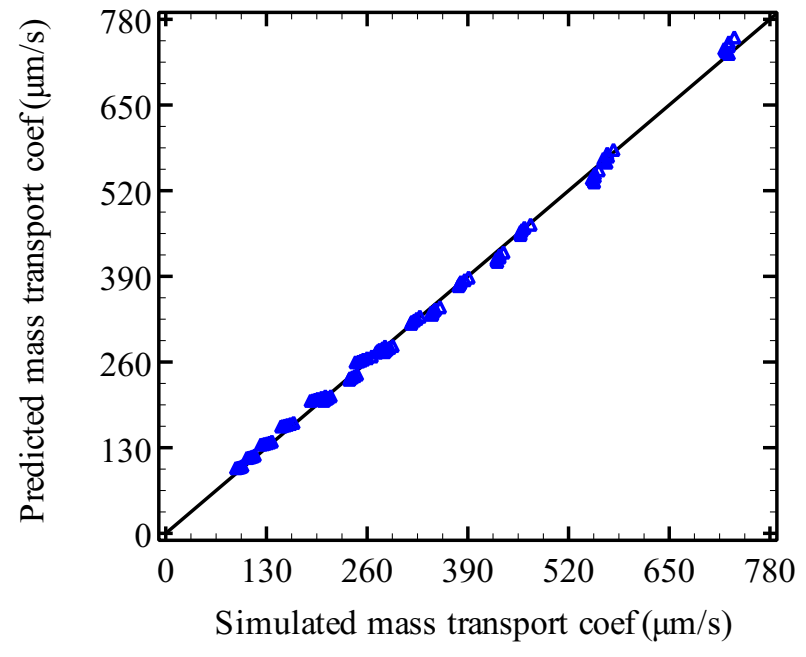

Figure 4: Comparison between simulated mass transport coefficient and the predicted one from equation (17) for the 108 simulations.

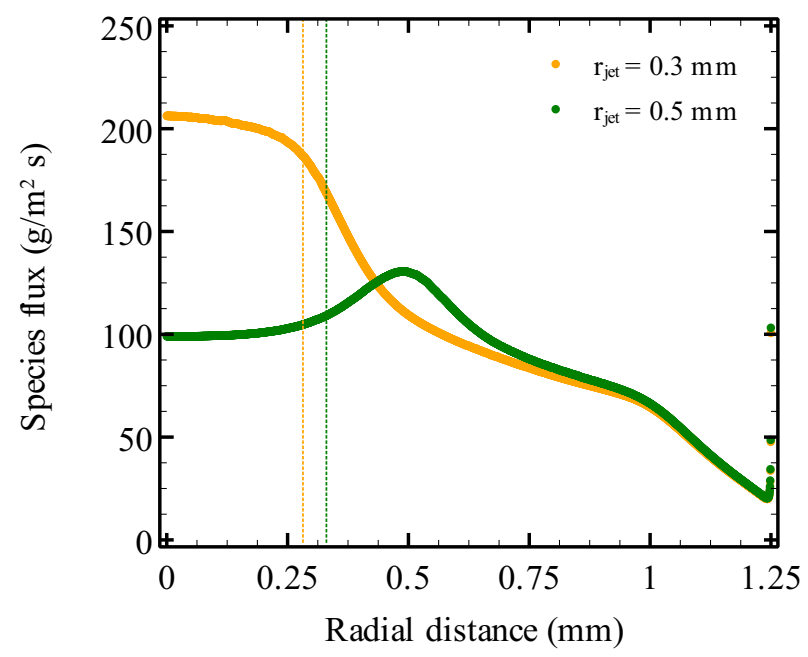

Figure 5: Simulated mass flux profiles at the electrode as a function of the radial distance from the center of the electrode for two geometries with different inlet radius at the same flow rate $(1 \mathrm{~mL} / \mathrm{min})$ where the radius of the electrode is $r_{\text {elec }}=1.25 \mathrm{~mm}$ : the curve in orange represents the mass flux where $r_{\text {jet }}=$ $0.3 \mathrm{~mm}$ and $h=0.15 \mathrm{~mm}$; the curve in green represents the mass flux where $r_{\text {jet }}=0.5 \mathrm{~mm}$ and $h=0.15 \mathrm{~mm}$; the dotted vertical lines represent the limits where the non homogeneity of species flux is below $10 \%$.

unlikely that consumption of the electroactive species further downstream has any influence on the concentration of species upstream. We have verified that this is the case in supplementary section S5.

Figure 5 shows the flux of species at the electrode as a function of the distance from the axis for two different simulations. The yellow curve (for which $r_{\text {jet }}=0.3 \mathrm{~mm}$ and $h=0.15 \mathrm{~mm}$ ) shows the typical behaviour observed in most of the simulations. The flux hardly changes between $r=0$, and a certain value, here $r \approx r_{\text {jet }}=0.3 \mathrm{~mm}$, and it then decreases with $r$. On the other hand, the green curve (for $r_{j e t}=0.5 \mathrm{~mm}$ ) is less common, but was found in a few simulations, especially 
at high values of $r_{j e t} / h$ ratio. In this case, after a region in which the flux varies little, it first increases significantly around $r=0.4 \mathrm{~mm}<r_{j e t}$, peaking about $r=r_{j e t}$ at a value $30 \%$ higher than the value at $r=0$ before decreasing slowly at higher values of $r$.

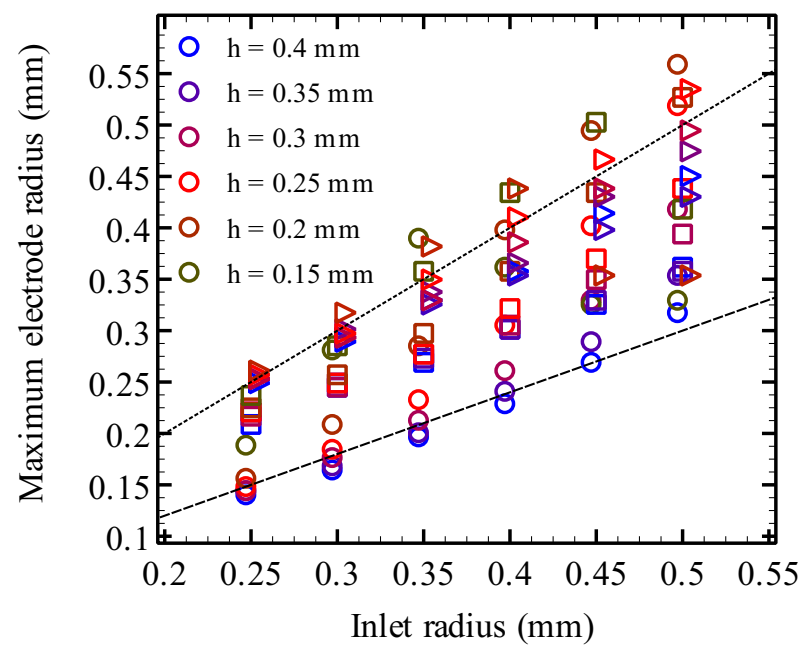

Figure 6: $r_{\max }$, the maximal value of the electrode radius for which the values of the flux for $0 \leq r \leq r_{\max }$ do not vary more than $10 \%$ as a function of the inlet radius, for different values of $h$ and $Q_{v}$. The values of $h$ can be deduced from the color of the symbol (see the legend inside the graph), while the shape of the symbols correspond to different values of $Q_{v}: Q_{v}=1 \mathrm{~mL} / \mathrm{min}$ for the circles, $Q_{v}=5 \mathrm{~mL} / \mathrm{min}$ for the squares and $Q_{v}=10 \mathrm{~mL} / \mathrm{min}$ for the triangles. The dot and the dash lines correspond respectively to $y=x$ and $y=0.6 x$.

For each of the simulations, we have determined the value $r_{\text {max }}$ for which the flux does not vary more than $10 \%$ between $r=0$ and $r=r_{\text {max }}$. The values are plotted in figure 6 as a function of $r_{j e t}$, and for the different values of $h$ and $Q_{v}$ used in the simulations. Overall, the points follow a linear relationship as a function of $r_{j e t}$, as is attested by the fact that they mostly lie between the $y=0.6 x$ and $y=x$ lines. In general, increasing the flow rate increases $r_{\text {max }}$ : the circles (for which $Q_{v}=1 \mathrm{~mL} / \mathrm{min}$ ) correspond to the lowest values of $r_{\text {max }}$, while the triangles $\left(Q_{v}=10 \mathrm{~mL} / \mathrm{min}\right)$ correspond to the highest values of $r_{\text {max }}$.

However, the individual dependence of $r_{\text {max }}$ on $r_{\text {jet }}$ can sometimes be more complex, as is attested by data for $h=0.15 \mathrm{~mm}$ and $Q_{v}=1 \mathrm{~mL} / \mathrm{min}$ in figure 6 . For $r_{\text {jet }} \leq 0.4 \mathrm{~mm}$, the value of $r_{\text {max }}$ increases with $r_{\text {jet }}$, but for larger values of $r_{\text {jet }}$, the value of $r_{\text {max }}$ decreases; this behaviour is related to the occurrence of the "atypical" behaviour discussed above in which the flux increases significantly about $r=r_{\text {jet }}$, before decreasing again (green curve in figure 5).

From the overall data in figure 6, we can conclude that the requirement that the variation in flux (or mass-transport coefficient) remains smaller than $10 \%$ is fulfilled when the radius of the electroactive area is smaller than $0.6 \times r_{j e t}$.

\subsection{Experimental validation}

To validate the relevance of the prediction of equation (10) in a real experimental setting, we built, using 3D-printing, a cell corresponding to $r_{j e t}=0.3 \mathrm{~mm}$ and $h=0.35 \mathrm{~mm}$. We performed cyclic voltammetry of a $\mathrm{K}_{3} \mathrm{Fe}(\mathrm{CN})_{6}$ solution for a number of flow rates. The results are plotted in figure 7 . Figure 7A shows the raw voltammograms, which show plateaus at low potential that are typical of a transport-limited reduction process. The fluctuations visible are linked to the argon flux necessary to keep the system as anaerobic as possible. From the values of the plateau current, it is possible to deduce the masstransport coefficient. We have plotted it against the power 0.45 of the flow rate in figure 7B (circles), along with the prediction of equation (10). The agreement is excellent, fully validating the whole approach.

\section{Discussion}

In this article, we have built upon our previous work, in which we have described a wall-tube cell designed for the studies of very fast enzymes[21]. Although we demonstrated that the cell was already improving upon the mass-transport properties of the RDE, which is commonly used for studying enzymes[1, 2], our previous work relied on CFD simulations for predicting the mass-transport properties of the cell, which is far too time- and resource-consuming for practical uses. Instead, for practical operation of the cell, and fine-tuning of its properties (geometric parameters), analytical formulas are required. The present work arises from attempts to i) validate the previously proposed formulas (or propose new ones) to accurately predict the mass-transport in our configuration; ii) verify the conditions under which the mass transport can be considered homogeneous and iii) propose formulas for predicting the shear stress, which has not been addressed in the literature before. The computation of the shear stress is important because it is likely that excessive shear stress could lead to washing of the enzymatic film[13], which has to be prevented as much as possible.

We used a two step approach to provide semi-empirical formula for predicting both the mass-transport coefficient and the shear stress values. First, with a coarse sensitivity study using a design of experiments approach applied to mass-transport values determined from simulations, we showed that only the flow rate, the jet radius and the distance between the inlet and the electrode have significant influence on the mass transport coefficient. Then, in a second approach, we use finer grained simulations with a greater number of parameter values to explore more systematically the parameter space. We used these data to propose semi-empirical formulas for both the mass-transport coefficient and the shear stress in the system. These formulas were able to reproduce simulated mass-transport coefficients and shear stress values with good accuracy, and were also very accurate to predict the experimental current obtained in a 3Dprinted cell (figure 7).

Table 4 summarizes some of the formulas available in the literature for the prediction of the mass-transport coefficient in wall-tube or wall-jet geometries, along with the smallest errors, the largest errors and the root-mean-squared deviation between the predicted value of mass transport and that measured on the 108 simulations of the second step of this work. For most of 


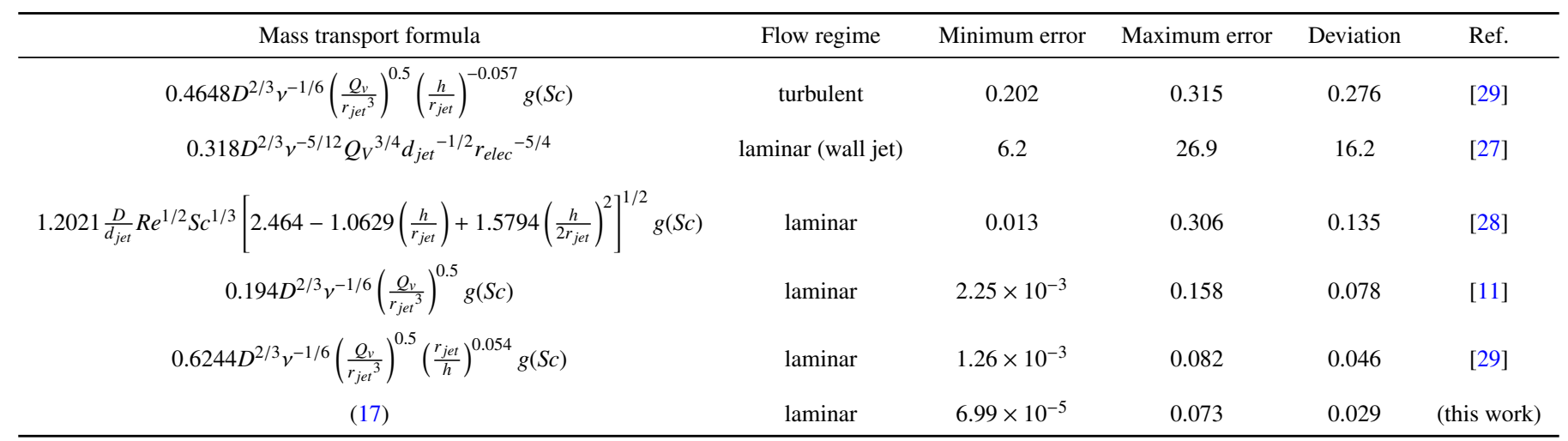

Table 4: Standard deviation and maximum deviation of the difference between the predicted and the simulated mass-transport coefficient for the case of the 108 simulations. $g(S c)$ is an asymptotic series whose development is: $g(S c)=1-0.084593 S c^{-1 / 3}-0.0016368 S c^{-2 / 3}-0.0057398 S c^{-1}+0.0014288 S c^{-3 / 4}+\ldots$

the formulas, the root-mean-squared deviation is in the order of $10 \%$ or significantly more, while the root-mean-squared deviation with respect to equation (17) is less than $3 \%$. Only the prediction of Chin and Tsang comes reasonably close to our proposition, with less than $5 \%$ error in average, and a maximum error not much above that of (17).

We could not find formulas that predict the wall shear stress in wall-jet and wall-tube electrodes in the literature, perhaps because the main objective of the published works is to predict the value of the current that one can obtain from a given experimental setup, but also probably because it is experimentally more challenging to measure the wall shear-stress, and it is hence harder to verify the given formulas experimentally. Rather than using an analytical formula to predict the shear stress, Esteban and coworkers use a combination of equations (10) and (12) to predict the shear stress from an experimentally determined mass-transport coefficient[12], in the context of corrosion studies. Here, we provide an analytical formula for predicting the shear stress. Although we could not validate our predictions for the shear stress experimentally, considering that both the mass-transport coefficient and the shear stress are derived from the same equations, based on the determination of the hydrodynamic constant $a$, we are confident that a good accuracy in the prediction of experimental mass-transport coefficients is an indication of the quality of the prediction of the shear stress too. It should be noted that, provided the equations (10) and (12) are valid (which is the case for both the wall-tube electrode and the $\mathrm{RDE}$ ), the shear stress is related to the mass-transport coefficient through the following formula:

$$
\tau_{w}=2.13 \times \frac{\rho v r}{D^{2}} \times m^{3}
$$

so that the shear stress scales as the cube of the mass transport coefficient. Therefore, the only way to greatly increase the mass-transport coefficient without a significant increase in the shear stress is to decrease the electrode size, since the shear stress is proportional to the distance from the rotational symmetry axis.

An important requirement to conduct protein film electrochemistry studies is the need for uniform accessibility: the masstransport should be homogeneous across the whole surface of the electrode, so that the concentration of substrate as perceived by all the molecules of enzymes is homogeneous. The rule-ofthumb found in the literature is that uniform accessibility is obtained in a wall-jet system when the electrode is smaller than the inlet[12]; this configuration is known as wall-tube. However, our data (figure 5) suggests that there can be significant heterogeneity even when the wall-tube condition is satisfied, and that for a more homogeneous mass-transport coefficient, a condition of $r_{\text {electrode }} \leq 0.6 \times r_{\text {jet }}$ is necessary. Our data also show that, under certain conditions, the center of the electrode does not correspond to the maximum of the mass transport coefficient, but that the transport increases slightly up to the size of the inlet, before decreasing (figure 5, green curve). MacPherson and coworkers experimentally observed a similar behaviour on a microjet electrode, which is similar to the setup we propose here, save for the smaller dimensions. Using electrodes significantly smaller than the inlet size, they could use a micropositioning device to map the mass transport coefficient as a function of the position of the electrode, and they also observed a slight increase when moving away from the center of the inlet[32].

Other works have emphasized the presence of recirculation patterns in wall-jet or wall-tube setups, in particular in the socalled "wall-jet" region, in which the radial velocity starts to decrease. These were predicted in some simulations [33], also in the case of the RDE[34], and observed experimentally in the case of the microjet electrode[35]. In the case of our simulations, we could not find traces of recirculation patterns in all the simulations (see for instance supplementary figure S10). Presumably, this is due to the fact that the horizontal flow proceeds in a restricted space of height $h$ comparable or even smaller than the inlet diameter, which leaves little space for recirculation patterns to occur.

Equation (17) shows that there cannot be an "optimal" setup, since the mass-transport coefficient can be made arbitrarily large by decreasing the size of the jet. A practical setup must therefore be a compromise between the mass-transport capacities obtained at a given flow rate and the practicality of building a cell with a small jet diameter, and an even smaller electrode diameter. The possibility to make very thin platinum electrodes were exploited by MacPherson and coworkers to build a micro- 

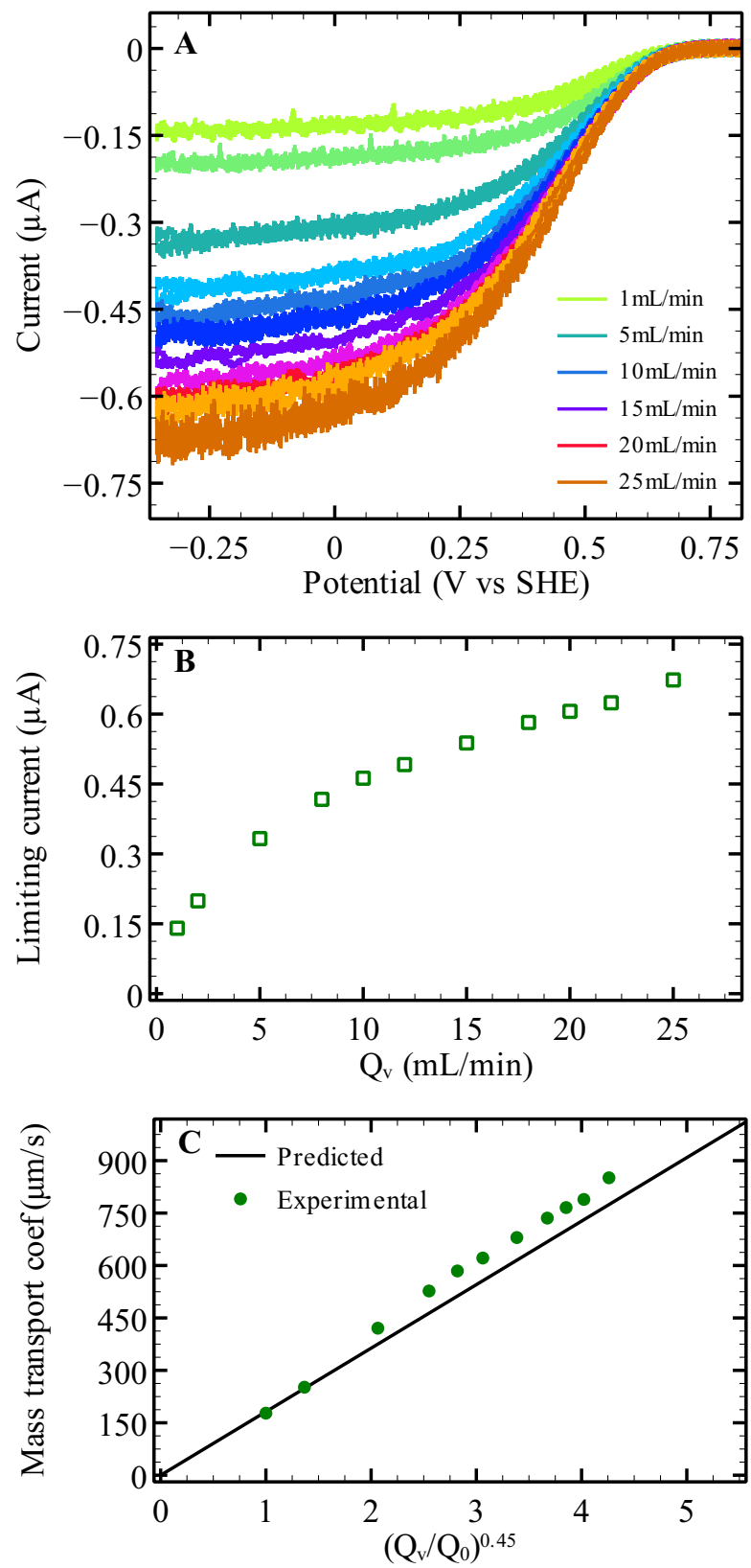

Figure 7: Experimental validation of the transport properties of one of the promising electrochemical cells $\left(r_{j e t}=0.3 \mathrm{~mm} ; h=0.35 \mathrm{~mm}\right.$ ): (A) Cyclic voltammetry for the reduction of $\mathrm{Fe}(\mathrm{CN})_{6}{ }^{3-}$ at a graphite electrode of diameter $100 \mu \mathrm{m}$ at $v=100 \mathrm{mV} . \mathrm{s}^{-1}$. The experiments were conducted at volume flow rate that ranged from $1 \mathrm{~mL} / \mathrm{min}$ to $25 \mathrm{~mL} / \mathrm{min}$ at room temperature, under degassed system with argon. (B) the limiting current (in absolute value) as a function of the flow rate. (C) Comparison of mass transport coefficient ( $m=I / n F A)$ extracted from the cyclic voltammetry experiments of the reduction of $\mathrm{Fe}(\mathrm{CN})_{6}{ }^{3-}$ (green circles) with the predicted mass transport coefficient from equation (17) (black line), $Q_{v}=1 \mathrm{~mL} / \mathrm{min}$. Conditions: $\left[\mathrm{Fe}(\mathrm{CN})_{6}{ }^{3-}\right]=1 \mathrm{mM}$, electrolyte: $0.5 \mathrm{M} \mathrm{NaCl}$, room temperature. We determined the working electrode's surface to be $8.2 \times 10^{-9} \mathrm{~m}^{2}$.

jet setup with an electrode size of $25 \mu \mathrm{m}$ and a nozzle diameter in the 80 to $120 \mu \mathrm{m}$ range, with the highest mass-transport reported to date with a wall jet system[36]. However, this sys- tem requires a horizontal micropositioning device to ensure the electrode is centered with respect to the inlet. With the application to PFE in mind, larger electrodes will have to be used, because the electrode of choice for PFE studies is the pyrolytic graphite edge electrode[2, 1, 7], which cannot easily be machined to sizes below $0.4 \mathrm{~mm}$. Together with the practical implications of using 3D-printed parts for the inlet, this sets a reasonable size of the inlet diameter around $0.6 \mathrm{~mm}$, which is what we have used in the experimental validation of figure 7 . However, the formulas and guidelines proposed in this article are likely valid for even smaller ranges, which means they could be used for much smaller electrodes.

The motivation of this work is to provide faster mass-transport than the setup usually employed for PFE studies, the RDE, as this setup, even used at very high rates, wasn't enough to prevent depletion for studying fast enzymes like the CO dehydrogenase[9]. For both the RDE and the wall-tube, the mass-transport is given by equation (10), with only the value of $a$ changing from one setup to the other. Therefore, the factor of increase of the transport over the RDE is given by:

$$
\frac{m_{W T}}{m_{R D E}}=\left(\frac{a_{W T}}{a_{R D E}}\right)^{1 / 2}=1.35 \frac{v^{0.04975} Q_{v}^{0.45025}}{h^{0.0345} r_{j e t}{ }^{1.41575} \omega^{1 / 2}}
$$

in which the quantities indexed with $R D E$ correspond to the RDE while those indexed with ${ }_{W T}$ refer to the wall-tube electrode. Assuming a reasonable maximum value of $5 \mathrm{krpm}$ for the rotation rate of the RDE, using the values of $r_{j e t}=0.5 \mathrm{~mm}$ and $h=0.35 \mathrm{~mm}$ and $Q_{v}=37 \mathrm{~mL} / \mathrm{min}$ as described in our previous work[21] yields the factor of 3 described previously. Using $r_{j e t}=0.3 \mathrm{~mm}$, the flow rate required for a factor-of-three improvement drops to $Q_{v}=10 \mathrm{~mL} / \mathrm{min}$. As was noted above (equation (18)), the wall-tube design in itself does not provide an advantage over the RDE with respect to the shear stress, since both setups share the same relationship between masstransport coefficient and shear-stress. However, while for the RDE, the only possibility for increasing mass-transport is to increase the rotation rate, but practical rates for rotating disc electrodes seldom go above $5 \mathrm{krpm}$. On the contrary, to increase the transport in the case of the wall tube electrode, one can either decrease the jet size or increase the flow rate, which provides more flexibility to achieve high mass transport coefficients.

\section{Acknowledgements}

The authors acknowledge support from CNRS, Aix-Marseille Université, Agence Nationale de la Recherche (ANR-15-CE050020, ANR-17-CE11-002), and the Excellence Initiative of AixMarseille University - A*MIDEX, a French "Investissements d'Avenir" programme (ANR-11-IDEX-0001-02), and the ANRDFG project SHIELDS (PL 746/2-1). They also wish to acknowledge expert help from Alain Grossi and Sady Noel in preparing the 3D-printed cell and the electrodes, and Gaëlle Coustillier and Philippe Delaporte from the laboratory "Lasers, Plasmas et Procédés Photoniques" in Marseille for their preparation of graphite samples of very small size, and finally Jérôme Vicente for insightful discussions and comments. AHA and VF are part of FrenchBIC (http://frenchbic.cnrs.fr). 


\section{Conflict of interest statement}

The authors have no conflict of interest to declare.

\section{References}

[1] C. Léger and P. Bertrand, Chemical Reviews, 2008, 108, 2379-2438.

[2] V. Fourmond and C. Léger, Adv. Biochem. Eng. Biotechnol., 2016, 158, $1-41$.

[3] M. del Barrio and V. Fourmond, ChemElectroChem, 2019, 6, 4949-4962.

[4] J. Hirst, Biochim. Biophys. Acta, 2006, 1757, 225-239.

[5] F. A. Armstrong, N. A. Belsey, J. A. Cracknell, G. Goldet, A. Parkin, E. Reisner, K. A. Vincent and A. F. Wait, Chem. Soc. Rev., 2009, 38, 36-51.

[6] T. Zeng, S. Leimkühler, U. Wollenberger and V. Fourmond, J. Am. Chem. Soc., 2017, 139, 11559-11567.

[7] F. A. Armstrong, H. A. Heering and J. Hirst, Chemical Society Reviews, 1997, 26, 169-179.

[8] M. Can, F. A. Armstrong and S. W. Ragsdale, Chemical reviews, 2014, 114, 4149-4174.

[9] M. Merrouch, J. Hadj-Saïd, C. Léger, S. Dementin and V. Fourmond, Electrochimica Acta, 2017, 245, 1059-1064.

[10] L. Domnik, M. Merrouch, S. Goetzl, J.-H. Jeoung, C. Léger, S. Dementin, V. Fourmond and H. Dobbek, Angewandte Chemie International Edition, 2017, 56, 15466-15469.

[11] W. J. Albery and S. Bruckenstein, Journal of Electroanalytical Chemistry and Interfacial Electrochemistry, 1983, 144, 105-112.

[12] J. Esteban, G. Hickey and M. Orazem, Corrosion, 1990, 46, 896-901.

[13] G. Binyamin and A. Heller, J. Electrochem. Soc., 1999, 146, 2965.

[14] S. G. Weber and W. C. Purdy, Analytical Chemistry, 1982, 54, 17571764.

[15] M. Glauert, Journal of Fluid Mechanics, 1956, 1, 625-643.

[16] J. Yamada and H. Matsuda, Journal of Electroanalytical Chemistry and Interfacial Electrochemistry, 1973, 44, 189-198.

[17] H. M. Elbardisy, E. M. Richter, R. D. Crapnell, M. P. Down, P. G. Gough, T. S. Belal, W. Talaat, H. G. Daabees and C. E. Banks, Analytical Methods, 2020, 12, 2152-2165.

[18] B. Fleet and C. Little, Journal of Chromatographic Science, 1974, 12 , $747-752$.

[19] J. L. Melville, B. A. Coles, R. G. Compton, N. Simjee, J. V. Macpherson and P. R. Unwin, The Journal of Physical Chemistry B, 2003, 107, 379386.

[20] W. J. Albery, Journal of electroanalytical chemistry and interfacial electrochemistry, 1985, 191, 1-13.

[21] M. Fadel, J.-V. Daurelle, V. Fourmond and J. Vicente, Physical Chemistry Chemical Physics, 2019, 21, 12360-12371.

[22] B. Levich, Acta Physicochim USSR, 1942, 17, 257.

[23] G. M. Carlomagno and A. Ianiro, Experimental thermal and fluid science, 2014, 58, 15-35.

[24] J. Seyed-Yagoobi, Drying technology, 1996, 14, 1173-1196.

[25] F. Homann, Forschg. Ing. Wes, 1936, 7, 1.

[26] C.-J. Chia, F. Giralt and O. Trass, Industrial $\mathcal{E}$ Engineering Chemistry Fundamentals, 1977, 16, 28-35.

[27] H. Matsuda and J. Yamada, Journal of Electroanalytical Chemistry and Interfacial Electrochemistry, 1971, 30, 261-270.

[28] M. T. Scholtz and O. Trass, AIChE Journal, 1970, 16, 82-90.

[29] D.-T. Chin and C.-H. Tsang, Journal of the Electrochemical Society, 1978, 125, 1461 .

[30] J. Antony, Design of experiments for engineers and scientists, Elsevier, 2014.

[31] N. Frossling, Lunds. Univ. Arsskr. NF Avd, 1940, 2, 35.

[32] J. V. Macpherson, M. A. Beeston and P. R. Unwin, J. Chem. Soc., Faraday Trans., 1995, 91, 899-904.

[33] J. Melville, N. Simjee, P. R. Unwin, B. A. Coles and R. G. Compton, J. Phys. Chem. B, 2002, 106, 2690-2698.

[34] A. Alexiadis, A. Cornell and M. Dudukovic, J. Electroanal. Chem., 2012, 669, 55-66.

[35] E. Bitziou, N. C. Rudd, M. A. Edwards and P. R. Unwin, Anal. Chem., 2006, 78, 1435-1443.

[36] J. V. Macpherson, S. Marcar and P. R. Unwin, Anal. Chem., 1994, 66 2175-2179. 